\section{Good for dentists, good for patients}

When The Dental Advisor tested Cavex Cream Alginate, it provided a 5-star rating.

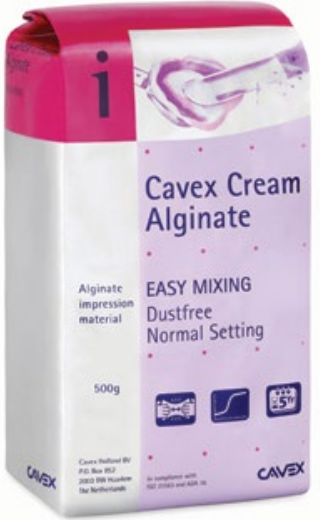

Dentists who have tried it talk about 'easy mixing' and 'good tear resistance'. It also offers 'excellent' detail and a short working time for the benefit of clinicians and patients alike. Less need for remakes means faster, more efficient treatment.

Cavex Cream Alginate is suitable for multiple castings and its high-detail replication enables digital impressions as well, making it a value product as well as a high-performance one.

Cavex Cream Alginate is distributed by J\&S Davis, as fast or normal set. Order direct or through your preferred dealer to see why it's the first choice for so many dentists.

For more information on the industry-leading products available from J\&S Davis, visit www.js-davis. co.uk, call 01438747344 or email jsdsales@js-davis.co.uk.

\title{
Don't let PPE waste become a new problem
}

With increased demand for singleuse PPE comes an increased amount of waste. Disposing of this waste responsibly is essential for ensuring the safety of others and the environment.

Initial Medical are experts in healthcare waste management and offer a full range of products, services and support to help dental practices optimise their waste management.
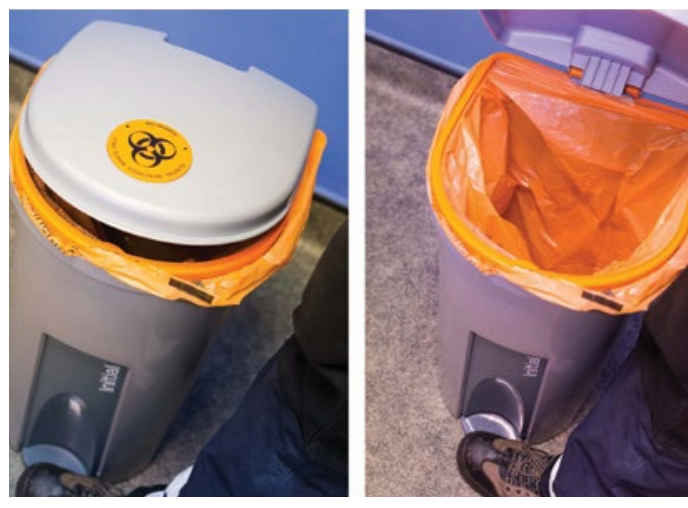

Initial Medical can help you create a dedicated PPE disposal area in your waiting room with their specifically designed $60 \mathrm{~L}$ capacity bags and SilverSafe, pedal-operated clinical bins. They are colour-coded orange to meet all legal recommendations and best practice protocols for the safe, effective and ethical disposal of potentially infectious waste. These are in addition to your normal clinical waste containers in the surgeries.

Initial Medical also offer a secure wheelie bin for lockable and fully-compliant storage, which it will collect at suitable intervals and ensure its safe disposal.

Ensure the appropriate disposal of PPE waste throughout your practice to stop it from becoming a new problem.

For further information visit www.initial.co.uk/medical or tel: 08708504045.

\section{Optimise your financial situation}

Given the events of 2020 so far, it has never been more important to stay on top of your finances and to optimise your situation wherever you can.

Right now, the significantly reduced interest rate - which is at an all-time low - affords opportunities to re-negotiate everything from

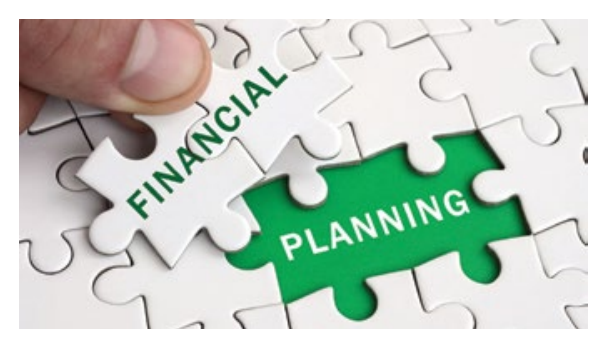

mortgage arrangements to loan repayments. Automating your banking and setting up direct debits could also help to streamline and vsimplify your expenses for better budgeting.

While making any changes, be wary of scams that tend to thrive when uncertainty and anxiety is high among the population, especially when it comes to managing your pension.

For peace of mind that you are truly optimising your finances, work with trusted IFAs, like the experienced team from money4dentists.

For more information call 0845345 5060, email info@money4dentists.com or visit www. money4dentists.com.

\section{Clean in-between}

Crisp packets, business cards, penknives - these are just some of the weird and wonderful things that patients have admitted using to remove debris from between their teeth.

But as well as risking infection and harm to gingiva, cleaning interdentally with such items can damage the teeth and cause abrasion-based wear on the enamel.

Tooth wear can cause a host of problems, both aesthetic and functional. Using a specially designed tool to clean between the teeth will remove the optimum amount of debris gently, yet efficiently.

Tandex interdental brushes, the FLEXI and the PROXI, come in a variety of sizes so are suitable for all patients. Easy to use and effective, they can fit into all the small spaces. In a variety of bold colours, they look good too. Used alongside Tandex GEL, which contains
$0.2 \%$ sodium fluoride, your patients will get a thorough, non-abrasive clean.

The FLEXI and PROXI are part of Tandex's range of high-quality oral health products, including toothbrushes and mouthwashes. Recommend Tandex to your patients - they'll never need reach for a credit card to clean their teeth again!

For more information on Tandex's range of products, visit www.tandex.dk. 